\title{
Effects of non-glide stresses on the plastic flow of single and polycrystals of molybdenum
}

\author{
V. Vitek ${ }^{\mathrm{a}, *}$, M. Mrovec ${ }^{\mathrm{a}}$, R. Gröger ${ }^{\mathrm{a}}$, J.L. Bassani ${ }^{\mathrm{b}}$, V. Racherla ${ }^{\mathrm{b}}$, L. Yin $^{\mathrm{b}}$ \\ ${ }^{\text {a }}$ Department of Materials Science and Engineering, University of Pennsylvania, Philadelphia, PA 19104, USA \\ ${ }^{\mathrm{b}}$ Department of Mechanical Engineering and Applied Mechanics, University of Pennsylvania, Philadelphia, PA 19104, USA
}

Received 26 September 2003; received in revised form 5 April 2004

\begin{abstract}
The Schmid law, which has been ubiquitously utilized in large-scale continuum computations, asserts that only the shear stress acting in the slip plane in the slip direction controls the plastic deformation. This rule is accurate for fcc metals but it does not apply in bcc metals owing to the spacial spreading of the core of screw dislocations that control their plastic deformation. In this paper we present resulsts of atomic-level simulations of the effect of externally applied stresses on 1/2[1 11 ] screw dislocations in molybdenum. We concentrate on the effect of other components of the applied stress tensor than the Schmid stress in the slip plane (10 1). These are shear stresses parallel to the Burgers vector in other $\left\{\begin{array}{lll}1 & 1 & 0\end{array}\right\}$ planes of the [ $\left.\begin{array}{lll}1 & 1 & 1\end{array}\right]$ zone as well as shear stresses perpendicular to the Burgers vector acting in $\left\{\begin{array}{llll}1 & 1 & 0\end{array}\right\}$ planes. We thus identify three distinct non-glide shear stresses that affect the glide of $1 / 2<111>$ screw dislocations and formulate single crystal yield criteria that include the effects of these stress components. This forms a basis for multislip yield criteria and flow relations for continuum analyses. Using this approach we demonstrate that the effects of non-glide stresses that originate at the level of individual dislocations have significant influence on plastic yielding of polycrystals.
\end{abstract}

(c) 2004 Elsevier B.V. All rights reserved.

Keywords: Peierls stress; Schmid law; Non-glide stress; Yield criterion; Multiple slip

\section{Introduction}

Continuum mechanics simulations of large plastic deformations are now becoming integral parts of both the design of components and the development of the manufacturing processes. The precursor of such calculations is a yield criterion that needs to be based on the physical understanding of the deformation properties of a given material. In crystalline materials such criterion has to be defined for each slip system, $\alpha$, and in a rate-independent formulation it can be written as:

$\Phi^{\alpha}(\sigma)=\Phi_{\text {critical }}^{\alpha}$

where $\Phi^{\alpha}$ is a yield function that depends, in general, on all the components of the applied stress tensor $\sigma$. The yielding commences when this function attains a critical value $\Phi_{\text {critical }}^{\alpha}$

* Corresponding author. Tel.: +1-215-898-7883; fax: +1-215-573-2128.

E-mail address: vitek@1rsm.upenn.edu (V. Vitek).
In most simulation codes the yield criterion has been based on the Schmid law [1-3] which asserts that glide on a given slip system is controlled solely by the resolved shear stress on that system, the Schmid stress, defined as:

$\tau^{\alpha}=n^{\alpha} \sigma m^{\alpha}$

where $n^{\alpha}$ is the slip plane normal and $m^{\alpha}$ the slip direction [4]. In this case the yield function is $\Phi^{\alpha}(\sigma)=\tau^{\alpha}$ and the yield criterion (1) states that glide on a slip system $\alpha$ commences when the Schmid stress $\tau^{\alpha}$ reaches a critical value. Implicitly, other components of the stress tensor and the sign of stress, play no role in the deformation process. However, it was pointed out already by Taylor and Elam in 1926 [5] that in metals like iron, which crystallizes in the body-centered-cubic structure (bcc), 'the mechanism of distortion is subject to laws which are quite different from those which govern the slip phenomena in any metal hitherto investigated'. The most prominent difference is the breakdown of the Schmid law manifested by the fact that the critical resolved shear stress (CRSS) for slip does not depend only on the Schmid stress but also on other components of the applied stress tensor. 
This aspect of the plastic behavior has been observed in a broad variety of bcc based metals and alloys [6-8] but not in close-packed crystals such as fcc metals. The most prominent difference between bcc and close-packed crystals is that in the latter case the crystal symmetry guarantees metastability of stacking faults on close-packed planes (see e.g. $[9,10]$ ), while the symmetry does not suggest any metastable stacking faults in bcc structure. Hence, in fcc and many hcp metals dislocations dissociate into Shockley partials connected by metastable stacking faults which keep the dislocations confined to the close-packed planes. In contrast, no such planar splitting exists in the bcc structure and the fact that [1 11 ] ] is the direction of a three-fold screw axis suggests that $1 / 2\left[\begin{array}{lll}1 & 1 & 1\end{array}\right]$ screw dislocations retain such symmetry and are thus intrinsically non-planar, as first suggested by Hirsch [11]. It is now generally accepted that the non-planarity of the cores of screw dislocations is responsible for the dominant characteristics of the plastic deformation of bcc metals, in particular for the breakdown of the Schmid law [6-8].

Atomistic studies of screw dislocations that employed a wide variety of descriptions of interatomic interactions are the principal source of our understanding of the atomic structure of dislocation cores. These calculations all confirmed the non-planarity and the three-fold screw symmetry of the core [7,12-14]. However, two types of the core of the $1 / 2\left[\begin{array}{lll}1 & 1 & 1\end{array}\right]$ screw dislocation were discovered. The first

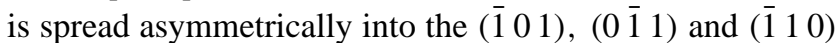
planes that belong to the [ $\left.\begin{array}{lll}1 & 1 & 1\end{array}\right]$ zone and is not invariant with respect to the [ $\left[\begin{array}{lll}1 & 0 & \overline{1}\end{array}\right]$ diad. Another energetically equivalent configuration related by this symmetry operation then exists and for this reason this core is called degenerate. The other core is invariant with respect to the [ $\left.\begin{array}{lll}1 & 0 & 1\end{array}\right]$ diad,

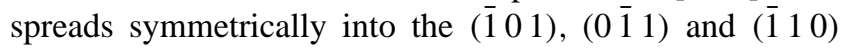
planes and is non-degenerate. Which aspects of bonding determine whether the former or the latter core structure is preferred has been discussed in a recent paper [14].

The atomistic studies revealed not only the structure of the core of $1 / 2\left[\begin{array}{lll}1 & 1 & 1\end{array}\right]$ screw dislocations but also the main reason for the breakdown of the Schmid law. Owing to the non-planar spreading, the core has to transform prior to the dislocation motion and this transformation may be affected by shear stresses in the slip direction acting in planes other than the slip plane, as well as by shear stresses in the direction perpendicular to the Burgers vector $[13,15,16]$. The break-down of the Schmid law then results from the impact of these non-glide stresses upon the dislocation motion via their influence on the dislocation cores. The most important outcome of atomistic studies is then finding the correct tensorial nature of stress-state dependence of the motion of dislocations. Using this result we can establish the dependence of the yield function on the applied stress tensor and project in this way the stress dependencies of the glide of individual dislocations into flow relations [14,17].

In this paper we present the results of atomistic studies of the glide of $1 / 2<111>$ screw dislocations in molybde- num performed using the recently developed bond-order potentials (BOP) that include physically correctly the mixed metallic and covalent character of bonding in this transition metal [18-20]. From the calculated dependence of the critical resolved shear stress (CRSS) on the orientation of the maximum resolved shear stress plane (MRSSP) we determine the shear stresses parallel to the Burgers vector that control the dislocation motion and enter the yield function. The next step is investigation of the dislocation glide when applying tensions and compressions with various loading axes. This, linked with the study of the combined effect of shear stresses perpendicular and parallel to the Burgers vector, determines the shear stresses perpendicular to the Burgers vector that have to be included into the yield.

Using these results we construct the yield criterion that reproduces accurately the stress-state dependence of the yield stress (Peierls stress) found in the atomistic calculations following refs. [21,22]. For the case of pure shear, this criterion is then applied in a Taylor-type calculation [23] which demonstrates that a significant non-Schmid effect, including a tension-compression asymmetry, results not only in single crystals but also in polycrystals.

\section{Motion of screw dislocations in molybdenum under the effect of shear stresses parallel to the Burgers vector}

The computer modeling of the $1 / 2\left[\begin{array}{lll}1 & 1 & 1\end{array}\right]$ screw dislocation and its glide was performed in the same way as in earlier calculations $[13,16]$. Periodic boundary conditions were imposed parallel to the dislocation line while perpendicular to the dislocation the block consisted of an active region, in which all the atoms were fully relaxed, and an inactive region where the atoms are permanently displaced in accordance with the anisotropic elastic field of the dislocation. When an external stress is applied, strain field corresponding to this stress is linearly superimposed. The active region contained 720 atoms and the inactive region 860 atoms.

The core structure found in this calculation is nondegenerate which is in agreement with recent DFT based ab initio calculations $[24,25]$. However, it the stress-state dependence of the Peierls stress that is of principal importance for physically correct formulation of the flow rules. When applying the shear stress parallel to the Burgers vector, the orientation of a MRSSP is defined by the angle $\chi$ which this plane makes with the slip plane $(\overline{1} 01)$. Owing to the crystal symmetry it is sufficient to consider $-30^{\circ} \leq \chi \leq+30^{\circ}$. In the following, we always give the shear stress acting in the [1 111 ] direction as the stress in the MRSSP. Its value when the dislocation starts to move, denoted $\tau_{\mathrm{M}}$, is identified with the CRSS for the dislocation glide at $0 \mathrm{~K}$, i.e. the Peierls stress.

The calculated dependence of $\tau_{M}$ on $\chi$ is displayed in Fig. 1. Interestingly, this dependence is not very different from that found for the degenerate core [16]. In both cases the Schmid law, according to which this dependence 


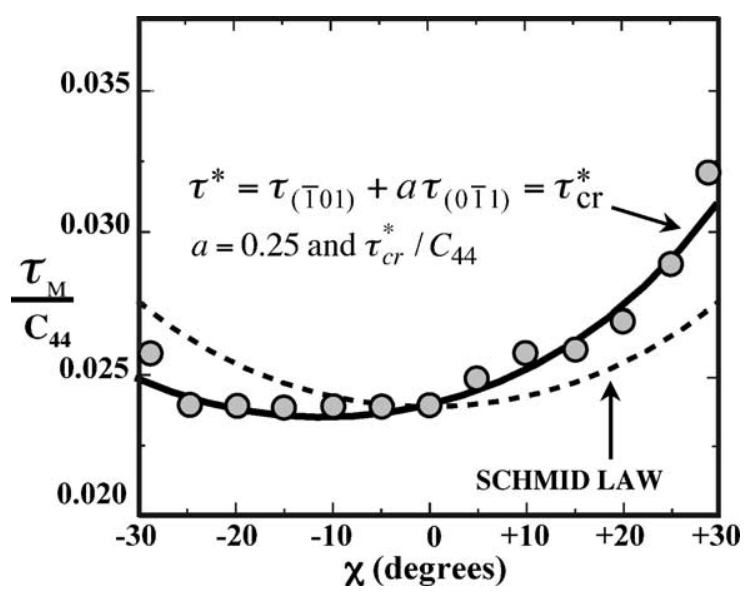

Fig. 1. Calculated dependence of the CRSS in the MRSSP, $\tau_{M}$, on the angle $\chi$ (circles). The dashed curve represents the Schmid law and the solid curve the yield criterion (3).

should have the form $1 / \cos \chi$ (the dashed curve in this figure), does not apply and $\tau_{\mathrm{M}}$ is larger for positive than for negative angles $\chi$; this corresponds to the so-called twinning-anti-twinning asymmetry [6]. Following the suggestion of Qin and Bassani [21,22] the effects of non-glide stresses may enter the flow rules for slip at the single crystal level by introducing an effective yield stress, $\tau^{* \alpha}$, which is a linear combination of the Schmid stress and other non-glide components of the stress tensor, that has to attain a critical value $\tau_{\mathrm{cr}}^{* \alpha}[17,26]$. For the pure shear in the direction of the Burgers vector, when the slip plane is ( 101$)$, the non-glide stress component can be identified with the shear stress acting parallel to the Burgers vector in the $(0 \overline{1} 1)$ plane. The full curve in Fig. 1 is based upon the yield criterion:

$\tau^{*}=\tau_{(\overline{1} 01)}+a \tau_{(0 \overline{1} 1)}=\tau_{\mathrm{cr}}^{*}$

with $a=0.25$ and $\tau_{\mathrm{cr}}^{*} / C_{44}=0.027 ; \tau_{\mathrm{M}}=\tau_{(\overline{1} 01)} / \cos (\chi)$.

\section{Application of tensile and compressive stresses}

The study of the motion of the 1/2[1 11 l] screw dislocation in molybdenum under the effect of applied tensions and compressions was performed analogously as in the case of pure shear (see also [16]). In each case the orientation of the loading axis within the standard triangle corresponds to an orientation of the MRSSP that can again be characterized by the angle $\chi$ defined above; of course, other components of the stress tensor than the shear stress in the direction of the Burgers vector are now present. If the dislocation motion were controlled solely by the shear stress parallel to the Burgers vector, the $\tau_{\mathrm{M}}$ would be the same for a given $\chi$, independent of the form of loading. This is not the case, similarly as in ref. [16], $\tau_{\mathrm{M}}$ varies with the orientation of the loading axis for a given $\chi$ and is different for tension and compression. This suggests that other components of the stress tensor than the shear parallel to the Burgers vector affect the CRSS significantly.

\section{Combined shear stresses parallel and perpendicular to the Burgers vector}

In order to decipher which stress components affect CRSS, it was proposed in [16] to investigate the influence of shear stresses perpendicular to the Burgers vector in a manner useful for understanding tension and compression. For a given MRSSP this is achieved by applying the stress tensor with the components $\sigma_{11}=-\tau_{2}, \sigma_{22}=\tau_{2}, \sigma_{33}=$ $\sigma_{12}=\sigma_{13}=\sigma_{23}=0$ in the right-handed coordinate system with the $x_{1}$ axis in the MRSSP, $x_{2}$ axis perpendicular to the MRSSP and $x_{3}$ axis parallel to [1 111$]$. In this way we attain shear stresses in the $\left\{\begin{array}{lll}1 & 1 & 0\end{array}\right\}$ planes of the [ $\left.\begin{array}{llll}1 & 1 & 1\end{array}\right]$ zone perpendicular to the Burgers vector and a direct link with the studies of tension and compression can be made. For example, for the loading axes [2 38 ] and [0 12 ] for which $\chi=0$, there is no shear stress perpendicular to the Burgers vector in the (1) 01$)$ plane, $-\tau_{2} \sqrt{3} / 2$ in the $(\overline{1} 10)$ and $\tau_{2} \sqrt{3} / 2$ in the $(0 \overline{1} 1)$ plane.

Fig. 2 shows the calculated dependence of the CRSS $\left(\tau_{\mathrm{M}}\right)$ on $\tau_{2}$ for $\chi=0$ when the stress tensor giving the shear stresses perpendicular to the Burgers vector was applied together with the shear stress parallel to the Burgers vector. It is important to note that changing the sign of $\tau_{2}$ corresponds to the rotation of the coordinates by $180^{\circ}$ around the [1 111$]$ axis. However, the core structure is not invariant with respect to this transformation and thus the effect of $\tau_{2}$ upon the dislocation behavior will, in general, be different for positive and negative values. The values of $\tau_{\mathrm{M}}$ corresponding to ten-

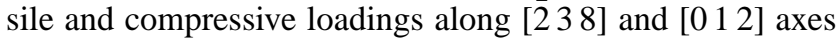
are also shown in this picture; for each uniaxial loading the value of $\tau_{2}$ is uniquely related to the loading stress at which the dislocation started to move. These values are obviously in close agreement with those obtained in the calculation

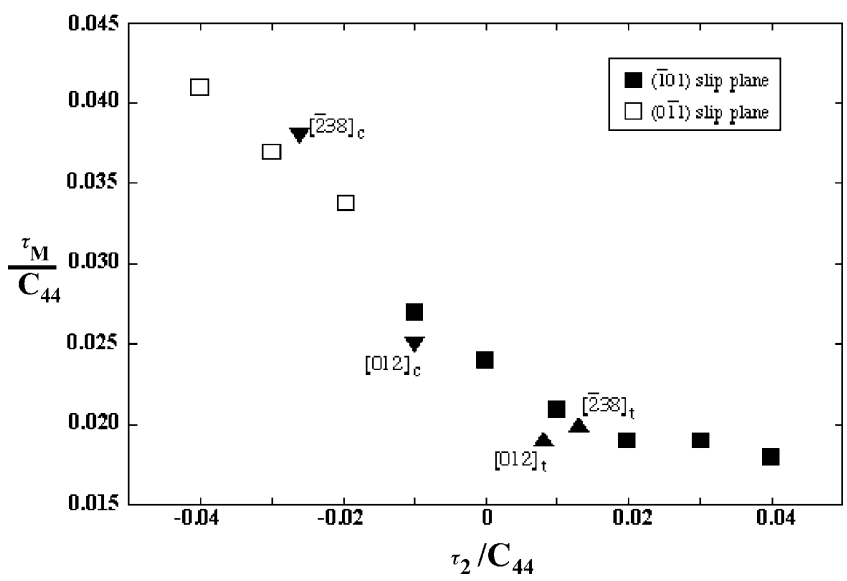

Fig. 2. Dependence of the CRSS ( $\left.\tau_{\mathrm{M}}\right)$ in the ( 10101$)$ plane $(\chi=0)$ on $\tau_{2}$ that defines the shear stress perpendicular to the Burgers vector. 
combining the stresses parallel and perpendicular to the Burgers vector independently. A similar result was obtained for loading axes that correspond to different orientations of the MRSSP and thus different angles $\chi$. This result demonstrates that the variation of $\tau_{\mathrm{M}}$ found for uniaxial loadings is governed by the shear stresses in the direction perpendicular to the Burgers vector that are represented by the deviatoric stress tensor defined above, which can then be expressed in terms of shear stresses perpendicular to the Burgers vector acting in two $\left\{\begin{array}{lll}1 & 1 & 0\end{array}\right\}$ planes of the [1 111$]$ zone.

\section{Continuum modeling of the plastic flow in molybdenum}

The results of the atomistic calculations presented in Sections 2 and 4 suggest that the glide of 1/2[lllll screw dislocations is governed by four distinct shear stress components. First two are the Schmid stress in the slip plane (1) 01 ) and the shear stress parallel to the Burgers vector in another $\left\{\begin{array}{lll}1 & 1 & 0\end{array}\right\}$ plane of the [llllll 111 zone. The other two are shear stresses perpendicular to the Burgers vector acting in two different $\left\{\begin{array}{lll}1 & 1 & 0\end{array}\right\}$ planes of the [1 111$]$ zone. Following the same philosophy as when deriving the yield criterion given by Eq. (3), the yield function can be written as a linear combination of these four shear stresses. The yield criterion for a slip system $\alpha$ is then:

$\tau^{* \alpha}=\tau^{\alpha}+\sum_{\eta=1}^{3} a_{\eta}^{\alpha} \tau_{\eta}^{\alpha}=\tau_{\mathrm{cr}}^{* \alpha}$

where $\tau^{\alpha}$ is the Schmid stress and $\tau_{\eta}^{\alpha}=n_{\eta}^{\alpha} \sigma m_{\eta}^{\alpha}$ are the non-glide stresses; $n_{\eta}^{\alpha}$ is the normal to the $\{110\}$ plane $\eta$, and $m_{\eta}^{\alpha}$ is the direction in this plane, either parallel or perpendicular to the Burgers vector, depending on which type of the shear stress is considered. In the case of the $1 / 2\left[\begin{array}{lll}1 & 1 & 1\end{array}\right]$ dislocation studied here the slip plane is $(\overline{1} 01) . \eta$ $=1$, related to a shear stress parallel to the Burgers vector, can be taken as corresponding to the $(0 \overline{1} 1)$ plane, similarly as in Eq. (3). $\eta=2$ and 3 then correspond to the shear stresses perpendicular to the Burgers vector in the planes

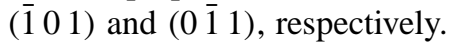

Calculation of the plastic deformation of a molybdenum single crystal loaded by an arbitrary applied stress requires consideration of all twelve slip systems of the $\left\{\begin{array}{lll}1 & 1 & 0\end{array}\right\}<\begin{array}{lll}1 & 1\end{array}$ > type. Since they are crystallographically equivalent they all obey the same yield criterion (4) but for a given slip system a distinction must be made between positive and negative stresses (and/or Burgers vectors). Using the Taylor homogenization procedure [23] we can analyze the plastic flow in a polycrystal where the yield criterion (4) is applied to every system in every grain.

We have studied uniaxially loaded random polycrystal but only shear stresses parallel to the Burgers vector were considered at this stage. In this case the yielding of each system is defined by an equation of the form Eq. (3), with $\tau_{\mathrm{cr}}^{* \alpha}$

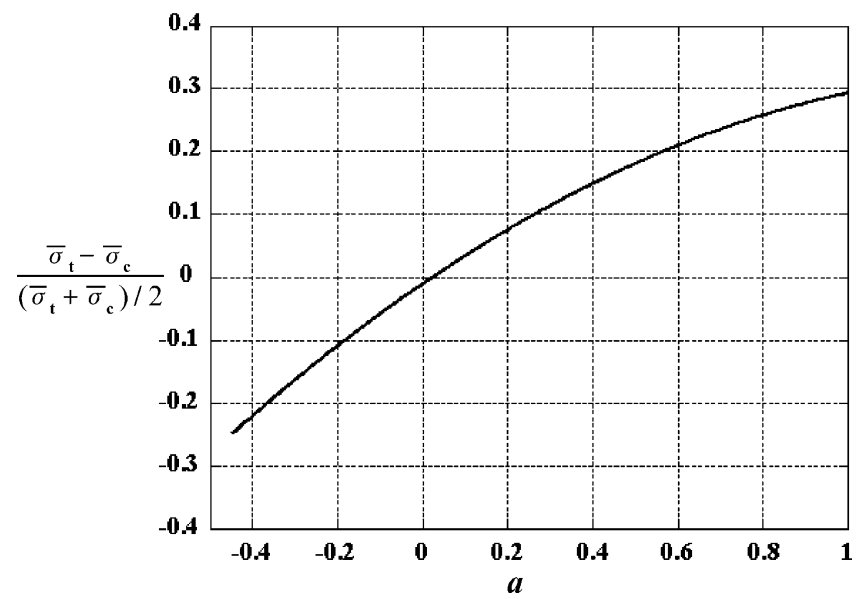

Fig. 3. Tension-compression asymmetry factor $\left(\bar{\sigma}_{\mathrm{t}}-\bar{\sigma}_{\mathrm{c}}\right) /\left[\left(\bar{\sigma}_{\mathrm{t}}+\bar{\sigma}_{\mathrm{c}}\right) / 2\right]$ for a random bcc polycrystal as a function of the 'strength' of the non-glide stress effect at the slip system level characterized by the coefficient $a$ defined by Eq. (3).

taken to be the same for each slip system. The most interesting is the tension-compression asymmetry that results from the effect of non-glide stresses upon the motion of individual dislocations and percolates to the plastic behavior of the random polycrystal. This is seen in Fig. 3 which shows the dependence of the tension-compression asymmetry factor $\left(\bar{\sigma}_{\mathrm{t}}-\bar{\sigma}_{\mathrm{c}}\right) /\left[\left(\bar{\sigma}_{\mathrm{t}}+\bar{\sigma}_{\mathrm{c}}\right) / 2\right]$, where $\bar{\sigma}_{\mathrm{t}}$ and $\bar{\sigma}_{\mathrm{c}}$ are macroscopic uniaxial tensile flow stresses in tension and compression respectively, upon the non-glide-stress parameter $a$, defined by Eq. (3). For molybdenum, studied in this paper, $a=0.25$ and the flow stress in tension is about $10 \%$ higher than the flow stress in compression.

\section{Conclusions}

Atomistic modeling of the effect of applied stresses of the $1 / 2\left[\begin{array}{ll}1 & 1\end{array}\right]$ screw dislocation revealed that there are four shear stress components that affect its glide. The first is the Schmid stress in the glide plane ( 1010 ) that drives its motion. However, the critical stress at which the glide commences also depends on the shear stress parallel to the Burgers vector

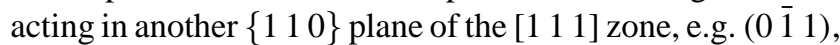
as well as on shear stresses perpendicular to the Burgers vector in the planes $(\overline{1} 01)$ and $(0 \overline{1} 1)$, respectively. The reason is that the core of the screw dislocation undergoes significant changes prior to the movement of the dislocation that are affected by the above mentioned non-glide stresses.

Using the results of these atomistic studies we proposed a physically-based continuum analysis of bcc crystal plasticity that incorporates the effects of non-glide shear components of the stress tensor via a generalized yield criterion employing the concept of an effective yield stress. This criterion has been incorporated into a full, three-dimensional continuum theory of multiple slip. The implications are not only striking at the single crystal level, but significant effects 
are also predicted at the polycrystalline level, specifically a prominent tension-compression asymmetry in a polycrystal with randomly oriented grains. Such effects are expected to be even more pronounced in textured polycrystals as well as in other materials with open structures in which dislocations are likely to possess non-planar cores.

\section{Acknowledgements}

This research was supported by the NSF Grant No. DMR02-19243.

\section{References}

[1] J.R. Rice, J. Mech. Phys. Sol. 19 (1971) 433.

[2] R. Hill, J.R. Rice, J. Mech. Phys. Sol. 20 (1972) 401.

[3] J.L. Bassani, Adv. Appl. Mech. 30 (1994) 191.

[4] E. Schmid, W. Boas, Kristallplastizität, Springer, Berlin, 1935.

[5] G.I. Taylor, C.F. Elam, Proc. R. Soc. London A 112 (1926) 337.

[6] J.W. Christian, Metall. Trans. A 14 (1983) 1237.

[7] M.S. Duesbery, in: F.R.N. Nabarro (Ed.), Dislocations in Solids, vol. 8, Elsevier, Amsterdam, 1989, p. 67.
[8] W. Pichl, Phys. Status Solidi (a) 189 (2002) 5.

[9] V. Vitek, Prog. Mater. Sci. 36 (1992) 1.

[10] V. Paidar, V. Vitek, in: J.H. Westbrook, R.L. Fleisher (Eds.), Intermetallic Compounds: Principles and Practice, vol. 3, Wiley, New York, 2002, p. 437.

[11] P.B. Hirsch, in: Proceedings of the 5th International Conference on Crystallography, Cambridge University, 1960, p. 139.

[12] V. Vitek, Cryst. Lattice Defects 5 (1974) 1.

[13] M.S. Duesbery, V. Vitek, Acta Mater. 46 (1998) 1481.

[14] V. Vitek, Philos. Mag. 84 (2004) 415.

[15] M.S. Duesbery, Proc. R. Soc. London A 392 (1984) 145.

[16] K. Ito, V. Vitek, Philos. Mag. A 81 (2001) 1387.

[17] J.L. Bassani, K. Ito, V. Vitek, Mater. Sci. Eng. A 319 (2001) 97.

[18] D.G. Pettifor, I.I. Oleinik, D. NguyenManh, V. Vitek, Comput. Mater. Sci. 23 (2002) 33.

[19] M. Mrovec, Bond-order potentials for BCC transition metals and molybdenum silicides, Ph.D. thesis, University of Pennsylvania, 2002.

[20] M. Mrovec, D. Nguyen-Manh, D.G. Pettifor, V. Vitek, Phys. Rev. B 69 (2004) 094115.

[21] Q. Qin, J.L. Bassani, J. Mech. Phys. Sol. 40 (1992) 813.

[22] Q. Qin, J.L. Bassani, J. Mech. Phys. Sol. 40 (1992) 835.

[23] G.I. Taylor, J. Inst. Met. 62 (1938) 307.

[24] C. Woodward, S.I. Rao, Philos. Mag. A 81 (2001) 1305.

[25] C. Woodward, S.I. Rao, Phys. Rev. Lett. 8821 (2002) 6402.

[26] V. Vitek, M. Mrovec, J.L. Bassani, Mater. Sci. Eng. A 365 (2004) 31 . 